\title{
Gestión sostenible de la energía en los hogares. Acciones colectivas en pos de más eficiencia y menos pobreza
}

\section{Sustainable household Energy management. Collective actions towards more efficiency and less poverty}

Raúl Zavalía Lagos, Guillermina Jacinto, Silvina Cecilia Carrizo, Salvador Gil raul.zavalia@fpvs.org,guillermina.jacinto@gmail.com,scarrizo@conicet.gov.ar, sgil@unsam.edu.ar

Fundación Pro Vivienda Social - Julio A. Roca 1341

San Miguel - Buenos Aires - Argentina;

CONICET - CESAL - Facultad de Ciencias Humana - Universidad Nacional del Centro de la

Provincia de Buenos Aires - Paraje Arroyo Seco s/n - Tandil - Buenos Aires - Argentina

CONICET - CIUT - Facultad de Arquitectura y Urbanismo - Universidad Nacional de La Plata - 47 nro. 162 La Plata - Buenos Aires - Argentina

Escuela de Ciencia y Tecnología Universidad Nacional de San Martín - Francia 34 - San

Martín Buenos Aires - Argentina

Recibido: 08/07/2020. Aceptado: 17/11/2020

\section{RESUMEN}

Acciones colectivas buscan un consumo de energía responsable y sostenible. Ellas movilizan la participación activa de usuarios y generar articulación de actores energéticos y territoriales. Conocer en qué se usa la energía, adoptar medidas de uso racional y eficiente, y procurar una gestión sostenible, puede contribuir a satisfacer las necesidades de la población, reducir sus consumos y sus gastos, sin perder calidad en sus servicios. Las reducciones en consumo de energía también alivian las asignaciones estatales en subsidios y mejoran la seguridad en la provisión de estos servicios. En Argentina, existen experiencias que promueven la inclusión energética, a partir de articular distintos actores y privilegiar el involucramiento de los usuarios de energía. Este trabajo tiene por objetivo mostrar estrategias que favorecen la gestión sostenible de la energía en los hogares, destacando la importancia que tiene en ello la capacitación de los vecinos y la organización social. Da cuenta de esto, a través de la presentación del proyecto Referentes Energéticos Barriales en el área metropolitana de Buenos Aires, que promueve auditorías energéticas como herramienta para facilitar el acceso al conocimiento, la gestión sostenible de la energía y el mejoramiento de la calidad de vida. Con él se busca realizar un aporte en la construcción de una sociedad más equitativa -con más eficiencia y menos pobreza- y a avanzar con los Objetivos de Desarrollo Sustentable, que el país se comprometió a cumplir.

Palabras clave: energía; eficiencia; inclusión social.

\begin{abstract}
Collective actions search for responsible and sustainable energy consumption. They mobilize the active participation of users and generate articulation of energy and territorial actors. Knowing what energy is used for, adopting measures for rational and efficient use, and seeking sustainable management, can contribute to satisfying the needs of the population,
\end{abstract}


reducing their consumption and expenses, without losing quality in their services. Reductions in energy consumption also alleviate state allocations in subsidies and improve security in the provision of these services. In Argentina, there are experiences that promote energy inclusion, by articulating different actors and encouraging the involvement of energy users. The objective of this paper is to show strategies that favor the sustainable management of energy in homes, emphasizing the importance of neighbors training and social organization. It gives an account of this through the presentation of the project Neighborhood Energy Advisors project in the Buenos Aires metropolitan area, that promotes energy audits as a tool to facilitate access to knowledge, sustainable energy management and life quality improvement. The project attempts to contribute to the construction of a more equitable society -with more efficiency and less poverty- and to make progress towards achieving the Sustainable Development Goals, which the country adopted.

Keywords: Energy; efficiency; social inclusion.

\section{INTRODUCCIÓN}

La energía es esencial para el bienestar de las sociedades y en el desarrollo humano. Disponer de energía no representa un fin en sí mismo, sino un vehículo que permite acceder a condiciones adecuadas de habitabilidad y lograr obtener servicios, tales como iluminación, transporte, calefacción, etc. El uso racional y eficiente de la energía (UREE) consiste en lograr estos servicios, usando la menor cantidad de energía posible. De este modo, se reduce el costo para satisfacer estas necesidades y se posibilita a más personas, acceder a estos servicios. Así, el UREE se convierte en una herramienta útil para ampliar la inclusión energética, mitigar la pobreza y las emisiones de gases efecto de invernadero.

La descarbonización de edificios es un gran desafío, y en particular los de viviendas (Sovacool, Martiskainen 2020). A su vez la pobreza y las privaciones energéticas ocupan un lugar destacado en la agenda de los gobiernos, con alcance e impactos considerables en sus presupuestos (Wilhite 2014). El uso racional de la energía y la utilización de equipos eficientes que una de las formas más rápidas de reducir las emisiones de gases efecto invernadero y de superar situaciones de déficit energético, permitiendo a la vez ahorrar energía y esfuerzos económicos (Tanides 2013). Luego se torna un factor clave para hacer viable y sostenible los servicios en hogares con bajos ingresos. Condiciones sociales, culturales y técnicas las dificultan también su posibilidad de aprovisionar energéticamente los hogares con servicios seguros y de calidad. (Durán 2018) La disponibilidad energía constituye un medio esencial para satisfacer las necesidades vitales y de confort humano. La pobreza energética afecta de formas diferentes a las poblaciones vulnerables (Carré 2013). Las políticas y condiciones socio-económicas que permitan acceder a los servicios energéticos. Frente al desafío de reducir las privaciones energéticas y buscando dar visibilidad a la necesidad de mejorar las condiciones del hábitat, la ONU plantea el Objetivo de Desarrollo Sostenible 7, de "garantizar el acceso a una energía asequible, segura, sostenible y moderna para todos". También la comunidad científica procura superar los enfoques exclusivamente teóricos o técnicos, y propiciar la adopción de soluciones apropiadas y co-construidas con las comunidades (Carrizo, Jacinto 2018). Estas responden a los desafíos de múltiples maneras, con nuevas propuestas de organización. La sociedad civil y los ciudadanos tienen un rol creciente en la definición e implementación de acciones e innovaciones que propicien su bienestar. Participan activamente y se involucran en la toma de decisiones y los proyectos territoriales (Gumuchian, 2003). 
En Argentina, la pobreza energética, como carencia de servicios energéticos adecuados, afecta a más de un tercio de la población. Estas personas no pueden satisfacer necesidades básicas, ni obtener condiciones mínimas de confort. La leña y el gas licuado de petróleo GLP (garrafas) son los combustibles a los que recurren las familias de bajos ingresos. Estos combustibles resultan entre 4-5 veces más caros que el gas natural por red. Por ende su adquisición impacta significativamente en sus presupuestos y además, la recolección de leña, demanda grandes esfuerzos físicos y compromete la salud de sus usuarios. (Jacinto, Carrizo y Gil, 2018)

La expresión popular "me llegó una factura enorme de gas o electricidad" revela una preocupación y un problema importante en la sociedad. En esa frase, el valor de la factura parecería un suceso fuera de control, casi un evento "natural" como una lluvia o un temporal, que está más allá del alcance de las acciones y decisiones de las familias. Esta expresión sugeriría que los ciudadanos no consideran sus posibilidades para gestionar los consumos energéticos. En cambio, las personas pueden conocer en qué usan y cómo consumen la energía, adoptar medidas de UREE y procurar una gestión más sostenible. Así podrían satisfacer mejor sus necesidades, reducir sus consumos y sus gastos, sin perder calidad en sus servicios. Para ello, es clave que los usuarios no permanezcan como actores pasivos, frente a las políticas energéticas o las decisiones empresariales, sino que comprendan cómo se usa la energía y cómo se la puede administrar. Las reducciones en consumo de energía también alivian las asignaciones estatales en subsidios y mejoran la seguridad en la provisión de estos servicios.

Acciones colectivas en esta búsqueda de un consumo responsable y sostenible, pueden movilizar la participación activa de los usuarios y generar articulación con otros actores involucrados energéticos y territoriales, como ser los municipios, ONG, distribuidores, etc. En Argentina, existen algunas experiencias embrionarias que están ganando visibilidad como las auditorías energéticas, promovidas desde la UNSAM Universidad Nacional de San Martín y organizadas a través de la Fundación Pro Vivienda Social (FPVS), con el apoyo de Edenor. (Fundación Pro Vivienda Social, 2019) Ellas facilitan el diagnóstico y modificación de los consumos residenciales, a través de referentes barriales capacitados y con participación de usuarios. Están siendo motores de cambio en barrios de bajos ingresos de la región metropolitana de Buenos Aires. Luego se abren interrogantes acerca de las posibilidades de conocer los consumos y de gestionar de forma sostenible la energía en los hogares, a la su vez que surgen preguntas sobre las oportunidades asociadas -de empleo, capacitación y otras- que también contribuyan a mejorar la calidad de vida de sus habitantes.

Este trabajo tiene por objetivo mostrar estrategias que favorecen la gestión sostenible de la energía en los hogares, destacando la importancia que tiene en ello la capacitación de los vecinos y la organización social. En particular se da cuenta de esto, a través de la presentación de una experiencia en curso, llevada adelante colectivamente por actores diversos, entre ellos ONG, Universidad, empresas privadas y profesionales y otros vecinos de distintas partes del país, que van captando el interés de la problemática y van sumando sus aportes.

\section{PERFIL ENERGÉTICO RESIDENCIAL EN ARGENTINA}

En 2018, los hogares argentinos consumieron 25\% de la energía producida en el país (Balances Energéticos, 2018). 5 consumos residenciales claves conforman alrededor del 80\% de ese cuarto: 1) acondicionamiento térmico de la vivienda (calefacción y aire acondicionado), 2) calentamiento de agua sanitaria ACS, 3) cocción, 4) refrigeración de alimentos e 5) iluminación. Aproximadamente, la calefacción y el calentamiento de agua sanitaria demandan 54\% y $34 \%$ del gas consumido en los hogares; $18 \%$ del consumo de gas es pasivo (llamas piloto) y podría reducirse o evitarse. La cocción representa $10 \%$ del 
consumo de gas residencial. La refrigeración de alimentos y el aire acondicionado absorben $38 \%$ y $20 \%$ de la electricidad usada en los hogares respectivamente. La iluminación se lleva $11 \%$. En cada servicio podrían realizarse grandes ahorros, modificando prácticas de consumo y usando tecnologías existentes y de bajo costo.

Referenciado los consumos de un hogar a los valores estándares de consumos eficientes, se podría determinar cuándo una vivienda excede el consumo esperable según sus características constructivas y región bioclimática. (Biloni et al., 2016) Luego eficientizar el hogar permitiría optimizar el consumo energético (ver recomendaciones).

La calidad de la envolvente es clave para el ahorro energético (Azqueta, 2017, 2018). Los equipamientos eficientes permitirían un ahorro del 50\% (o superior) en electricidad. Reducir los consumos a la mitad, significa reducir notablemente la factura energética porque disminuyendo los consumos se pasa a la categoría de usuario de electricidad y/o de gas, con menor cargo fijo y gasto variable. Por ejemplo, en el Gran Buenos Aires, consumir la mitad de la energía, podría significar una reducción factor 3,5 en los gastos de las familias. El uso de sistemas solares térmicos para el ACS permitiría reducir aún más los consumos de energía (Iannelli, 2016).

Para los grupos vulnerables y de menores ingresos, medidas de uso racional y eficiencia podrían significar la superación de situaciones de precariedad y pobreza energética. Nuevas formas de organización entre actores múltiples adaptan soluciones energéticas. Estas favorecen que familias dispongan de más recursos energéticos y económicos y que estos beneficios repercutan entre los actores involucrados. Se trata de procesos de intervención social colaborativos, en redes territoriales de acción. Así organizaciones comunitarias, Estado, instituciones académicas, empresas de energía y los propios usuarios, avanzan en la construcción de nuevos modelos de gestión de la energía.

\section{GESTIONAR LA ENERGÍA EN LOS HOGARES}

La Escuela de Ciencia y Tecnología de la Universidad Nacional de San Martín (UNSAM) y la Fundación Pro Vivienda Social implementan el Proyecto "Referentes Energéticos Barriales". Con las auditorías energéticas como herramienta, capacitan a los propios usuarios en la medición de sus consumos energéticos residenciales. La medición directa permite conocer cuantitativamente el consumo de la totalidad de los equipos funcionando en un hogar. A partir de esto pueden identificarse los consumos más importantes en una vivienda y graficarlos, como en la figura 4. Conocer los consumos residenciales más relevantes permite reflexionar sobre cuándo y cómo se usa la energía y diseñar acciones para optimizar los consumos y reducir las facturas.

\subsection{Referentes Energéticos Barriales}

El Proyecto Referentes Energéticos Barriales de la UNSAM-FPVS asocia dos estrategias, complementarias. La primera estrategia es capacitar estudiantes de la carrera de Ingeniería de la Energía (UNSAM) en la realización de auditorías energéticas residenciales. Los estudiantes capacitados relevaron los consumos energéticos en sus hogares, representativos de sectores socioeconómicos medios, con gas natural. Al 2020, más de 100 estudiantes recibieron la formación y realizaron las auditorías. La segunda estrategia consiste en capacitar referentes energéticos barriales, principalmente miembros de la Fundación Pro Vivienda Social (FPVS). Son vecinos de los municipios de Moreno y Pilar (Provincia de Buenos Aires) que residen en barrios de bajos ingresos económicos. Aprenden a realizar auditorías energéticas, pautas para hacer un uso eficiente de la energía en las viviendas. Esto lo instrumentan en sus hogares y barrios. 
Consumo Energético - RA 2018

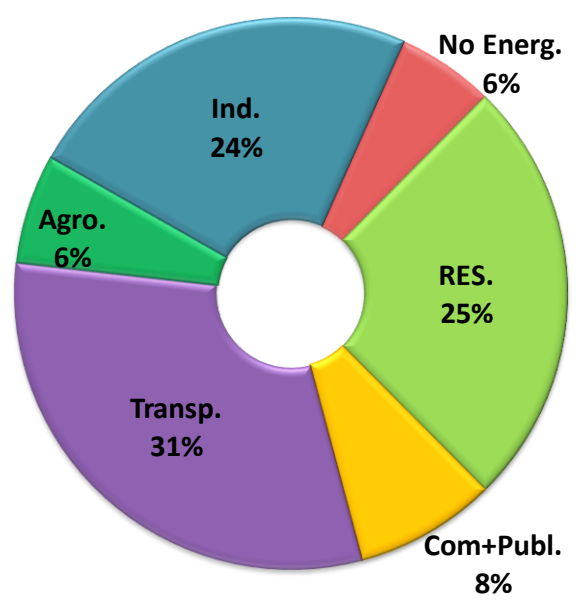

\section{Consumo Eléctrico 2018}

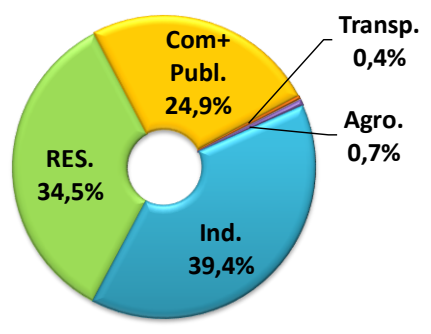

Gas Natural Año=2018

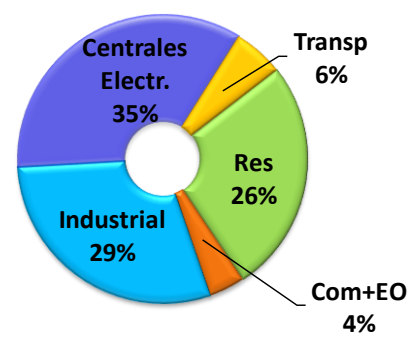

Figuras 1. Izquierda, consumo energético total en Argentina al año 2018. El consumo residencial es el 25\% del consumo total. A la derecha, distribución del consumo eléctrico y de gas natural para el mismo año. (Balances Energéticos, 2018), (ENARGAS - Natural Gas Regulatory Agency of Argentina, 2020), (Instituto Nacional de Estadística y Censos, 2010).

En un trabajo colaborativo, los referentes energéticos realizaron auditorías en sus hogares, con asistencia y asesoramiento de los estudiantes y docentes de la UNSAM. Esos referentes barriales se apropiaron del "know how" y continuaron realizando más auditorías en las viviendas de sus vecinos y capacitando a otros. En este proceso los vecinos comienzan a conocer y administrar sus consumos y a adoptar medidas de UREE. Los resultados obtenidos también fueron de utilidad para la distribuidora eléctrica, la cual decidió financiar buena parte de estas auditorías. A su vez, sirvieron para retroalimentar el proceso de capacitación, a lo largo del cual se revisaron y discutieron los datos, en reuniones periódicas, realizadas en la Universidad. Para el sector académico, disponer de datos de consumo resulta valioso para la generación de conocimiento, que contribuya a delinear mejor propuestas fundamentadas de políticas públicas.

Se concretaron 89 auditorías energéticas en barrios de Moreno y Pilar. A partir de los datos relevados, los referentes barriales realizaron devoluciones personalizadas a cada hogar, orientadas a la adopción de medidas de UREE. Además, conociendo sus consumos, muchos vecinos decidieron regularizar las conexiones eléctricas de sus viviendas mediante la instalación de medidores autoadministrados o prepagos, denominados MIDE Medidor Integrado de Energía.

\subsection{Anatomía de los consumos residenciales metropolitanos}

Las auditorías energéticas realizadas permitieron conocer la anatomía de los consumos residenciales, como se ve en la figura 2. En los hogares de ingresos medios, en vivienda con servicio de gas por redes, los consumos claves -calefacción, calentamiento de agua sanitaria, cocción, aire acondicionado e iluminación- constituyen más del $88 \%$ del total. El principal consumo eléctrico es la preservación de alimentos (heladeras). En los hogares de ingresos bajos, sin acceso a gas por red, los consumos claves -cocción, calentamiento de agua sanitaria, 
calefacción eléctrica, heladera e iluminación- implican $76 \%$ del total. El principal consumo eléctrico es el agua caliente sanitaria (ACS).

Consumo total prom. $=17,8 \mathrm{MWh} / \mathrm{año}$

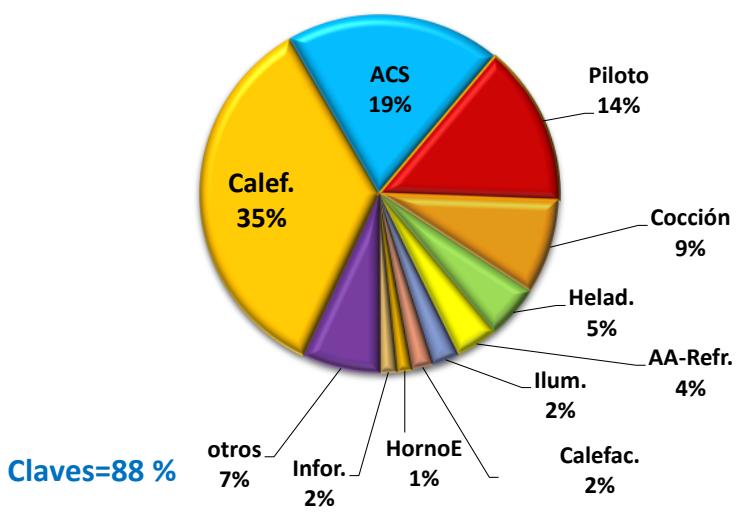

\section{Consumo Total Medio=8,48 MWh/año}

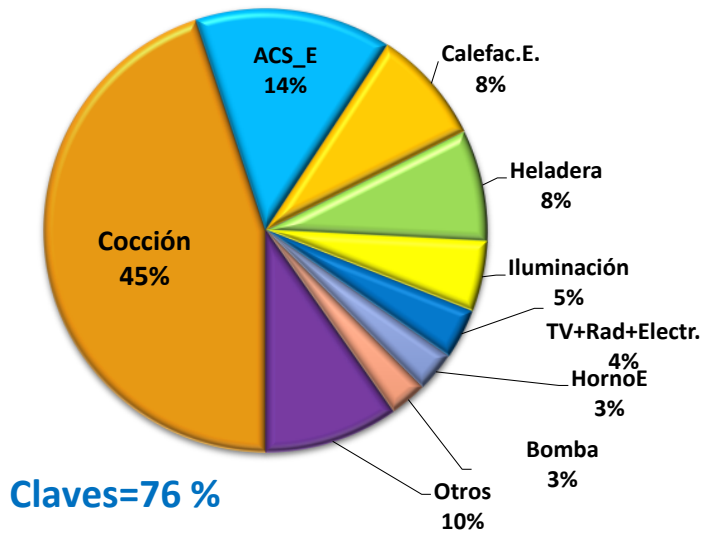

Figura 2. Distribución del consumo energético residencial. A la izquierda para viviendas de ingresos medios, con servicios de gas por redes. A la derecha, distribución del consumo eléctrico residencial en viviendas de bajos ingresos, sin acceso a gas natural por redes. Elaborado a partir de auditorías realizadas en casas de 99 estudiantes de la UNSAM y en 89 casas de Villa Rosa, de Pilar, Provincia de Buenos Aires.

En relación a los consumos totales en viviendas de bajos ingresos, se observa una gran dispersión, como se ilustra en la figura 3. Allí se separan en cuatro cuartiles los consumos y se ve que el consumo medio de cada cuartil es bien diferente. Es notable que el primer cuartil de usuarios consume un cuarto de energía, respecto del promedio; mientras que el último cuartil usa cuatro veces más energía que el promedio. Por otra parte, entre el primer cuartil y el cuarto hay un factor 22 en el uso de la energía.

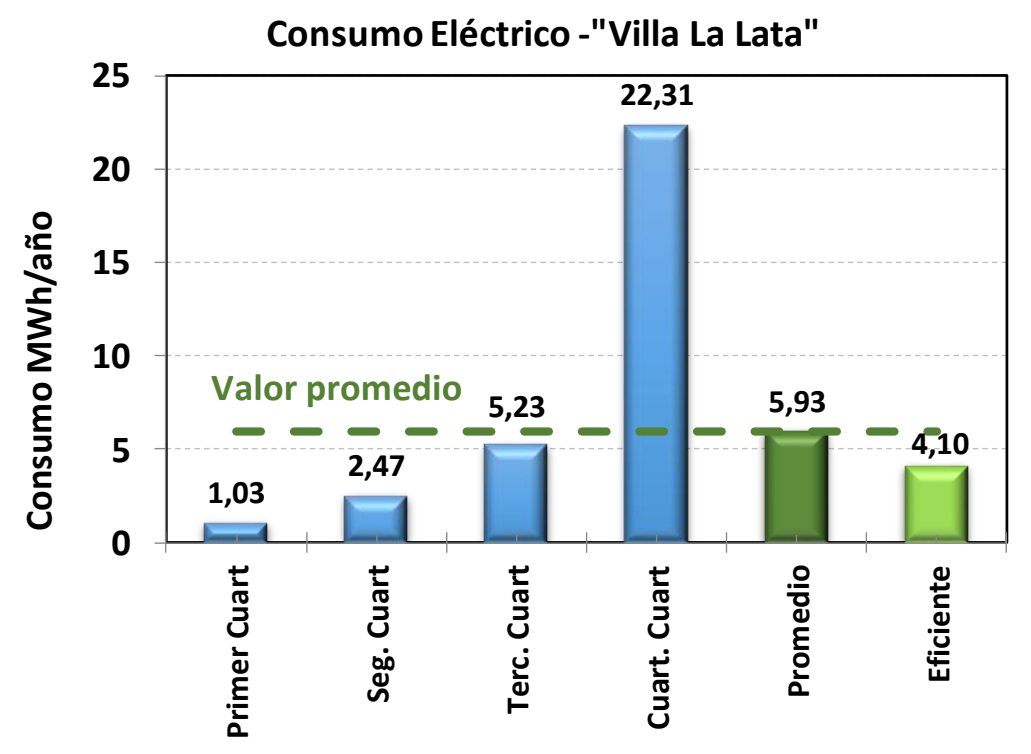

Figura 3. Consumo específico eléctrico residencial de 99 viviendas auditadas en Proyecto Referentes Barriales, separado en cuatro cuartiles y comparado al consumo promedio de la muestra y al consumo estimado para un usuario que hace un uso eficiente de los recursos 
Los consumos residenciales pueden reducirse con medidas de UREE. Como se ve en la figura 4 , tanto los hogares de ingresos medios como los de bajos recursos, ambos pueden obtener ahorros del orden del 50\%. Si los consumos se redujeran en factor de 2, el gasto monetario en energía se reduciría en un factor mucho mayor, ya que al bajar los consumos se pasa a categoría de usuarios con menor cargo fijo y gasto variable en la factura de electricidad y/o de gas. En la región del Gran Buenos Aires y en la Ciudad de Buenos Aires, una reducción de un factor 2 en el consumo de energía, puede implicar una reducción de 3,5 en los gastos de energía de las familias.

\section{Consumo Eficiente Tot.= 8,2 MWh/año}

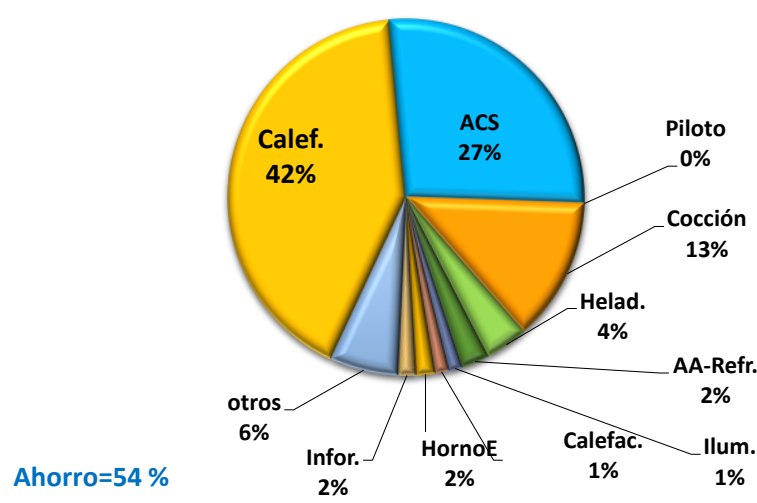

\section{Consumo Total Eff=4,79 MWh/año}

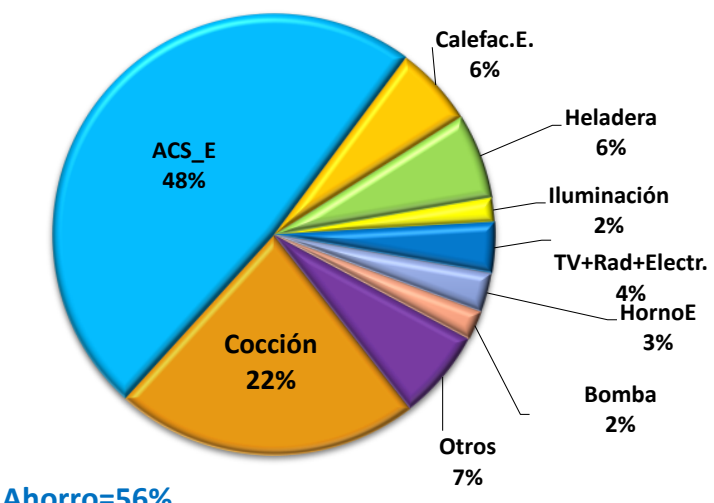

Figuras 4. Distribución del consumo energético residencial eficiente. A la izquierda para viviendas de ingresos medios, con servicios de gas por redes. A la derecha, distribución del eléctrico residencial, viviendas de bajos recursos, sin acceso a gas natural por redes. Elaborado a partir de auditorías realizadas en casas de 99 estudiantes de la UNSAM y en 89 casas de Villa Rosa, de Pilar, Provincia de Buenos Aires. Como se ve en ambos casos con medidas de UREE es posible reducir los consumos en el orden del $50 \%$.

Comparando los consumos obtenidos para cada tipo de vivienda y los consumos estimados para una vivienda eficiente, los Referentes Energéticos Barriales pueden identificar dónde aplicar medidas de eficiencia energética y asesorar a los vecinos. Capitalizando la experiencia del Proyecto, la Escuela de Ciencia y Tecnología de la UNSAM, desarrolla una Diplomatura (90 horas) para continuar y ampliar la formación de Referentes Energéticos Barriales.

\section{Discusión}

La instrumentación de las medidas recomendadas permite disminuir los consumos, sin deteriorar su calidad de vida o mejorándola. Con ello, no sólo se beneficia el vecino, que reduce consumos y gastos, sino además el Estado, que puede reducir el presupuesto en subsidios energéticos y se cuida el ambiente disminuyendo las emisiones de gases de efecto de invernadero (GEI). De esta manera, se avanza hacia escenarios de mayor responsabilidad e involucramiento de los usuarios en la gestión de la energía. 


\section{CONCLUSIONES}

En las viviendas de Argentina existe un grupo de 5 a 7 servicios energéticos claves implican alrededor de tres cuartos del consumo residencial. Los principales consumos son 1) acondicionamiento térmico de la vivienda (calefacción y aire acondicionado), 2) calentamiento de agua sanitaria ACS, 3) cocción, 4) preservación de alimentos (heladeras) e 5) iluminación. Focalizando en acciones de UREE en estos servicios, es posible disminuirlos considerablemente, que es una estrategia crucial para procurar una gestión sostenible de la energía en los hogares, en particular en los de menores ingresos.

Las auditorías energéticas en manos de referentes barriales son una herramienta potente para descifrar y modificar los consumos residenciales. Los referentes energéticos facilitan la realización de un análisis cuantitativo de los consumos. Con ellos hacen diagnósticos precisos y confiables a cada vecino y son capaces de recomendar estrategias de UREE, específicas para cada familia. La eficiencia de los equipos (principalmente calefón/termotanque y heladeras) y la aislación de la envolvente de las viviendas, son fundamentales para reducir los consumos, las facturas energéticas y los gastos del Estado, en subsidios a la energía. No menos importante, es que la promoción de equipos eficientes ayudaría a reactivar la industria nacional.

Políticas públicas que estimulen el acceso y la adopción masiva de artefactos eficientes, favorecería una reducción considerable en el costo de las facturas energéticas y que un mayor número de personas acceda a los beneficios de la energía. En ese sentido, acciones de trabajo que articulan distintos actores y privilegian el involucramiento de los usuarios de energía, promueven la inclusión energética. Así las auditorías energéticas resultan una herramienta que facilita el acceso al conocimiento y a la gestión sostenible de la energía, para mejorar la calidad de vida de vastos grupos de población. Capacitar Referentes Energéticos Barriales y al conjunto de los vecinos en prácticas de UREE, mejoraría las posibilidades laborales de muchas personas, en trabajos asociados a la sostenibilidad. Con ello, se tiende a construir una sociedad más equitativa -con más eficiencia y menos pobreza- y a avanzar con los Objetivos de Desarrollo Sustentable, que el país se comprometió a cumplir.

\section{RECOMENDACIONES}

Entre las modificaciones sugeridas parar eficientizar el hogar se encuentran:

- $\quad$ Mejorar la aislación de la envolvente (paredes, techos y aberturas)

- Reducir las infiltraciones de aire con cintas de carpintero y nylon

- Calefaccionar de manera adecuada y eficiente

- Regular la temperatura de calefacción/refrigeración (termostato)

- Adoptar sistemas ACS y electrodomésticos de etiqueta A (o superior) en eficiencia

- eliminar los consumos pasivos (pilotos)

- adoptar tecnologías de iluminación LED

\section{REFERENCIAS}

AZQUETA, P. (2018). Una mirada al aislamiento térmico y la eficiencia energética. Hojitas IEDS CNEA. p. 197.

AZQUETA P. (2017). «Manual Práctico del Aislamiento Térmico en la Construcción,» AAPE 2nda. Edición, Buenos Aires. 
BALANCES ENERGÉTICOS. (2018). Recuperado el 7 de abril de 2020 http://datos.minem.gob.ar/dataset/balances-energeticos

BILONI J., COBAS ANDRINOLO, M. B.; CARRIZO, S., CODESEIRA, L., FIORA, J., GASTIARENA, M.; IANNELlI L., JACINTO, G.; PRIETO, R.; GIL, S. (2016) Sostenibilidad y eficiencia en el suministro de servicios energéticos a poblaciones dispersas y de bajos recursos. Energías Renovables y Medio Ambiente, 38, 15 - 23

CARRE, M. N. et Grupo (2013). «Vulnerabilités energétiques urbaines dans les Amériques». Confins, $\mathrm{N}^{\circ} 18$. https://confins.revues.org/8380

CARRIZO, S., JACINTO G. LORENZO P., GIL S. (2019) Sostenibilidad y eficiencia en el suministro de servicios energéticos a poblaciones dispersas y de bajos recursos: pobreza energética /. - 1a ed. - Ciudad Autónoma de Buenos Aires: FODECO, 60p.

CARRIZO, S., JACINTO G. (2018) Co-construcciones de redes energéticas. Acciones colectivas territoriales en Argentina, siglo XXI. Confins 35 https://journals.openedition.org/confins/12988

DURAN, R. (2018). Apuntes sobre pobreza energética: estimaciones para Argentina: año 2003-2018. In: BERTINAT P.; MORERO B.; CHEMES J. (ed.) Taller Ecologista. Rosario.

ENARGAS - Ente Nacional Regulador del Gas. (2020). ENARGAS. Recuperado el 5 de marzo 2020 de https://www.enargas.gob.ar/

FUNDACIÓN PRO VIVIENDA SOCIAL. (2019). Fundación Pro Vivienda Social (FPVS). Recuperado el 5 de mayo 2020 de http://fpvs.org/.

GUMUCHIAN, H. (2003). Les acteurs, ces oubliés du territoire, Paris: Anthropos, 186 p.

IANNELLI, L., PRIETO, R., GIL, S., (2016). Eficiencia en el calentamiento de agua. Consumos pasivos en sistemas convencionales y solares híbridos. Petrotecnia, LV (3), 586-595.

INSTITUTO NACIONAL DE ESTADÍSTICA Y CENSOS (INDEC). (2010). Censo Nacional de Población, Hogares y Viviendas 2010. Recuperado el 3 de junio 2020, de https://www.indec.gob.ar/

JACINTO, G., CARRIZO, S., y GIL, S. (2018). Pobreza energética en Argentina. Ideas para servicios sostenibles en el Norte de Argentino. Petrotecnia, LVII 3/18, 26-30.

LIU E., LAGISZ M., JUDD B. (2019). Policy and program options for improving energy efficiency in low income households - Rapid systematic review of evidence. CRCLCL, Sydney, Australia.

SOVACOOL B. K., MARTISKAINEN M. (2020). Hot transformations: Governing rapid and deep household heating transitions in China, Denmark, Finland and the United Kingdom. Energy Policy 139111330.

TANÍDES, C. et al. (2013). Escenarios energéticos para la Argentina (2013-2030) con políticas de eficiencia, Buenos Aires: Fundación Vida Silvestre.

WILHITE, H. (2014). "Insights from social practice and social learning Theory for sustainable energy consumption", Flux, Paris, n.96/2, 24-30 